\title{
Information of Public Security Traffic cloud platform framework
}

\author{
Sang XueKun \\ Information Center, China Waterborne Transport \\ Research Institute \\ China Waterborne Transport Research Institute \\ Beijing, China \\ e-mail: sxk8238@139.com
}

\author{
Ma WeiWei \\ Information Center, China Waterborne Transport \\ Research Institute \\ China Waterborne Transport Research Institute \\ Beijing, China \\ e-mail: baijing@wti.ac.cn
}

\begin{abstract}
With the rapid development of Internet technology, cloud services technology as a solution to deal with the complexity of distributed computing applications and software development environment is a hot technology is gradually being adopted in all walks of life. Traffic public security industry as a transportation hub for intelligence information, also need to cloud services technology in traffic management in the public security characteristics of integrated information, Through the Internet exchange and sharing the transport Public Security Bureau personnel, vehicles, cargo, ships, logistics, information, Throughout the internal transport Public Security Bureau Public Security industry consolidation of the transport characteristics of each group information database to virtualization, resource, building people, vehicles and goods, boats, etc. Information of Public Security Traffic cloud platform framework, the public security sector to resolve internal traffic Features intelligence information island phenomenon of increasingly serious problem of intelligence information resources scale difficult to play, to achieve the greatest degree of public security close to the actual transport, port and shipping services, the goal of public service.
\end{abstract}

Keywords- cloud services technology; transport public security; virtualization; Close combat; resources

\section{INTRODUCTION}

2014 National Conference on Transportation, Minister Yang Chuantang requirements of the current and future periods to fully deepen reform, concentrating accelerate the "four traffic" development, one of which four traffic "integrated transport" mentioned basic conditions and national strategies, respond to the new demands of new industrialization, informatization, urbanization, agricultural modernization simultaneous development, overall planning of railways, highways, waterways, civil aviation and postal industry, the establishment of institutional mechanisms for improvement and integrated transport system to adapt, improve service levels, logistics efficiency and overall effectiveness.

The complexity of the current domestic and international security situation, facing a huge incoming traffic, logistics, information flow and personnel flow, cross-harbor water transport logistics and more serious crimes, water security situation being challenged for incoming public security "hit, anti-, Control "also presents practical challenges. Therefore, we must vigorously strengthen the information technology, the integration of traditional incoming transport system resources and technology to enhance public security intelligent transportation "playing, prevention and control," the ability and level.

\section{Transportation PUblic SECURITy FEATURES BACKGROUND INFORMATION CLOUD PLATFORM}

A. Traffic is restricted public security information technology development, integration and innovation urgently needed

"Eleventh Five-Year" and "five" A few years ago all incoming traffic on the public security bureau of information technology, has invested a lot of manpower, financial and material resources, the establishment of a large number of business applications, has accumulated a lot of basic information, for the safe transport of incoming played a significant role. However, with the economic globalization, the spread of information society, the traditional single-dispersed wading Hong Kongrelated personnel, vehicles, ships, cargo and other management has been unable to meet the new situation of water transport safety. Mainly in the wading Hong Kongrelated personnel, vehicles, ships, cargo and other information is scattered, isolated data storage, low level of information sharing, a serious shortage of information resources development and utilization, overall economies of scale do not come out to play. How to integrate existing resources, sustainable economic development for the incoming raised new challenges.

As incoming traffic safety administration, in order to change the traditional management practices, and achieve close combat, service incoming targets, it must be scientific information to guide incoming public security information. Comprehensive assessment of the current level of development of information incoming traffic police, showing a good momentum of development in general, but in the use of integrated and value-added services personnel, vehicles, ships, cargo transport these characteristic elements of public security information is not enough, incoming traffic public security services, business management decision-making ability is relatively weak, the overall efficiency and economies of scale 
incoming administration of public security does not come out to play. Thus, through the establishment of a vertical and horizontal integration, collaborative interaction incoming police officers, vehicles, ships, cargo characteristics of integrated information cloud platform is imperative.

B. cloud services technology innovation to lead the future health of the rapid development of new trends in incoming police

Cloud services technology, you can either keep the independence of the Public Security Bureau of existing ports and shipping units of the Public Security Bureau and other wading Hong Kong-related business application systems, integrity, and they can break the closure of existing business application systems, [3]open up "islands of information" between channels, to establish a unified cloud platform virtualization traffic public security people, vehicles, vessels, etc. characteristics of information and achieve integration cloud resource utilization and request access to, thereby increasing the degree of public security traffic characteristics shared information resources. In this context, the cloud platform for public security concerns Traffic incoming personnel, vehicles, ships, cargo and other characteristics of information technology-based cloud services, the use of modern technology to achieve incoming traffic for wading involving Hong Kong's public security "people, vehicles and goods "and" people, boats, cargo, "a comprehensive high-end use, to break the bottleneck of the development of water transport, port and shipping management to achieve" five-second "period digital, intelligent management and low-carbon, green and sustainable development.

\section{THE CLOUD PLATFORM FRAMEWORK RESEARCH AND DESIGN}

Traditional information traffic incoming Public Security Bureau, Public Security Bureau and other units shipping is confined to the unit for providing services, and a new generation of "digital incoming" is characterized by a high degree of integration and utilization of information resources, incoming transport chain each node are seamlessly perfect combination. Incoming units role in the entire supply chain is weakened, all incoming units internal information resources of great social and economic value will be strengthened up. Information flow integration, providing services for all incoming units loosely coupled, differentiated, personalized service.

A hot cloud service is a distributed application environment approach to complex operations and software development solutions. This is characterized by loosely coupled services that can better adapt to changing business environment, such as changing business level, business focus. This kind of cloud services-based architecture provides a simple and practical ways and means of application integration for the realization of distributed application systems integration and interoperability of information silos possible. [5]
Features information on cloud services platform to build traffic police officers, vehicles, ships, cargo and other cloud-based services technology, through the development of a unified interface standards and interface specifications of the traditional application of some of the transport PSB slight transformation, the original of the transport PSB incoming service virtualization, cloud area became a theoretical level.

Traffic police officers, vehicles, ships, cargo, physical characteristic information cloud platform frame structure shown in Figure 1.

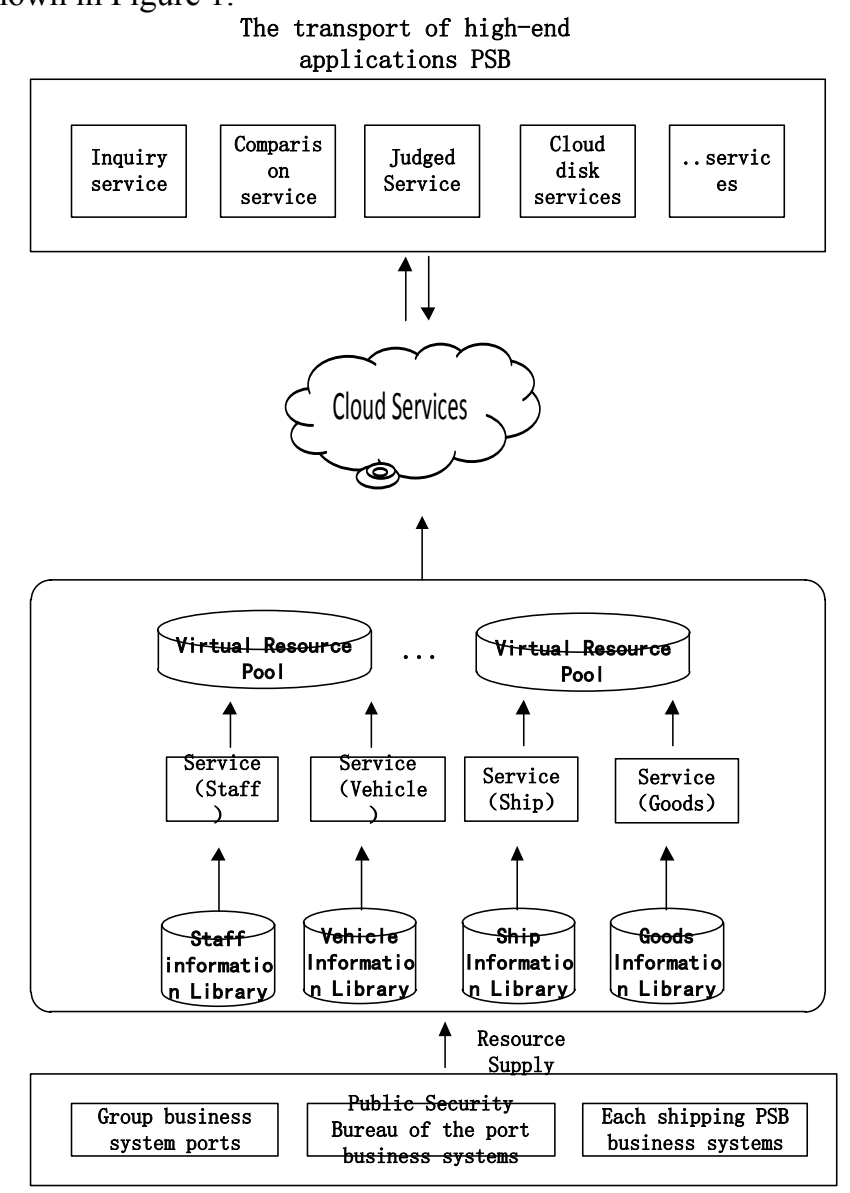

Figure1. A traffic information Public Security Features Figure cloud platform framework

Can be seen from the figure, the entire traffic police officers, vehicles, ships, cargo and other special information cloud platforms, including the supply of resources, resource storage virtualization, cloud services, high-end applications constitute, respectively, as follows:

- resource supply: an important part of the entire cloud platform, the main provider of public security, and specialty transportation sources and gathering information, the information currently featured traffic police officers, vehicles, ships, cargo and other major ports in the relevant group from the production system, the Public Security Bureau of the port-related business systems, as well as the Public Security Bureau of shipping related business systems, each bureau by establishing an interface with the relevant system, the first time the Council convergence of information resources. 
- virtualized resources Storage: Storage is virtualized resources Key part of the whole cloud platform, virtualization resource is the Public Security Bureau of the transport characteristics of the information gathered to re planning to establish pools of virtualized resources, including personnel, vehicles, vessels, cargo and other information virtualized resource pools for the whole cloud platform services.

- Cloud Services: Cloud services are a core part of the platform, which is the business applications across the entire platform framework request hub, users can apply for business applications through the cloud platform agency services, cloud services according to certain calculations, seeking and response services request, and give compliance feedback.

- High-End applications: the overall framework of the uppermost cloud platform, through the establishment of public security of the entire transport data request service standards available to the Public Security Bureau Traffic end cloud services, cloud-end applications to achieve a real sense.

\section{THE RELEVANT TECHNICAL RESEARCH}

Transportation Public Security Features achieve business information platform for the cloud will face many design and technical issues, therefore, the public security during the transport characteristics of business information platform for research and design of the cloud, you first need to study several related technologies, as follows:

\section{A. Data gathering techniques}

Research data aggregation technology is mainly related to the dynamic information of each port group, port Public Security Bureau, Public Security Bureau personnel shipping related business systems, vehicles, vessels, cargo, etc. aggregation, primarily incoming entire transportation supply chain efficient data extraction out, and then follow the thematic categories, the business class, the base class respectively converge to form a single branch of valid data repository, each branch of the reunification of converging to a large repository of various types of data.

\section{B. Virtual Storage Technology}

Virtual storage technology resources mainly through relatively mature virtualization technology (hardware) for the classification of data aggregation virtual storage resources, and establish resource directory and data resources backup mechanism to ensure the safety of fresh and data resources.

\section{Cloud computing technology}

Cloud computing and virtualization technology is used in conjunction with each other in a technology platform in the research and design of the framework, the main advantage of cloud computing technology to achieve the overall service provided cloud platform, using the best algorithms for high-traffic public security application provides a virtual cloud structures.

\section{Request services}

Request service technology is a relatively mature technology, the research and design of the platform framework, the main content and request permission to access the research service object acquired on the basis of analysis of some of the current application, tap the true sense of the cloud platform query cloud, cloud comparison, judged cloud, cloud storage, high-end applications.

\section{E. Under a cloud computing environment} Transportation Service of Public Security standard research platform technology

Traffic Public Security resource virtualization technology specification standards and structures, as well as service-oriented architecture objects, capture platform access standard cloud adaptation framework and standards. Each of the main building, operation and maintenance of standards to protect research platform to co-ordinate the transport Public Security Bureau, Port Group, maritime, water, etc., to fully mobilize the forces of the market, to build traffic information Public Security cloud platform.

Public Security Bureau to develop the transportoriented cloud services platform specifications, build a resource-access devices and cloud computing system adapted each Port Group, Maritime Bureau, Water Board, the establishment of resource access to high-performance computing devices and application software, building facing Public Security Bureau of the transport characteristics of cloud services platform, to achieve the full range of open traffic police information interactively query service.

\section{The Ultimate GOAL}

Study of Public Security to carry out traffic information cloud platform framework, notably services, the solution is efficient, will achieve the following objectives. [1]

\section{A. The establishment of Public Security Traffic Information Service System}

Cloud computing is based on the latest science and the use of modern techniques, the integration of existing internal distribution of traffic across the public security bureau of people, cars, boats, cargo, and material data clusters, using its virtualization, networking, and the formation of the overall resource pool, according to the development trend of modern management and fourth generation port, the port is completed fully digital, eventually forming cloud service system of public security traffic characteristics information.

\section{B. Information sharing and business collaboration through an efficient system of public security}

Cloud services platform to solve water transportation industry information convergence poor public security issues, the service platform to pursue accurate, timely, comprehensive and user-friendly information, by combing business content and processes on a regular basis to provide intelligent solutions. 


\section{To achieve efficient use of resources}

Framework established between cloud services platform sharing and dynamic allocation of resources in the transport Public Security Bureau data, so that can be dynamically scalable, easier to manage, and improve the utilization of the overall system security and resources to address the Ministry of Public Security Bureau and the traffic the degree of utilization of resources.

\section{Management of change breakthrough}

Framework through the cloud computing platform from the application software to hardware, storage, and network construction, the traditional IT architecture model has now been transformed into virtual mode will eventually achieve a unified cloud computing architecture model.

\section{CONCLUSION}

Cloud technology and mature development of virtualization technology for the application of all walks of life has brought considerable economic benefits and a lot of new management ideas, cloud computing to "like water, electricity, gas and other public utility services, like access to a computer over a network the ability to "show people, the most prominent advantage is to make information technology to" turn on the lights "as easy. For the overall utilization and processing industry data, the project technology and flexible expandability make investments in information technology industry is greatly reduced.

So the application of cloud technology and virtualization technology to the utilization of the public security traffic characteristics of business information is a bold attempt by the current Research and Design of information of Public Security traffic cloud platform framework, the theory is to meet the transport needs of the public security sector characteristics, and be able to provide some business support role in the follow-up work will also use the platform design framework for the actual project development, and improvement in the running for the public security traffic close combat, service port and shipping services, in order to better play a positive role for the incoming traffic of transport safety.[7]

\section{REFERENCES}

[1] Zhao MingJiang, "Port Integrated Information Framework cloud services platform,” vol 2 ,Beijing China, June 2011.

[2] Zhang Jian, Cloud computing concept and influence to resolve LJ, Telecommunications network technology, 2009, pp.15-18.

[3] Xie SiJiang and Feng Yan, "Analysis of Cloud Computing and Information Security, Beijing University of Posts and Telecommunications," Beijing Institute of Electronic Science and Technology, 2008.

[4] Li Ye, "Development of cloud computing" Beijing University of Posts and Telecommunications.2011.

[5] Qin YaLing, "Based research activities in the design and application of Google cloud services," Shanghai Normal University, 2010.

[6] Liu YuXiang, Reflections on the Public Security Information toplevel design," Public Security Education, 2010.

[7] Hu ShiMin, Lead to the construction of public security information of Modern Police, Shanghai Public Security College, 2009.

[8] Shen Xiang,EPublic security information technology to solve basic problems, Wuhan Public Security Cadre Institute,2009.

[9] Lu Shan, "Request Broker Research Services and its application in a distributed system of public security," Hunan University, Dec. 2005, pp. 73-99.

[10] Sun Liang, "The fourth generation port features and port logistics development trend of China [J], 2009[1],pp40-55. 